\title{
miR-548ac Inhibit Wnt5a Expression and Promotes Proliferation of Glioma Cells through the AKT/ERK Pathway
}

\section{Wen Shan}

Affiliated Hospital of Nantong University https://orcid.org/0000-0002-2795-7625

Haonan Li

Affiliated Hospital of Nantong University

\section{Xu Lu}

Affiliated Hospital of Nantong University

\section{Xuting Wu}

Shandong University Qilu Hospital

Xinhua Zhang ( $\nabla$ zhangxinhua@ntu.edu.cn )

Nantong University https://orcid.org/0000-0002-5702-6733

\section{Primary research}

Keywords: miRNAs, Cell Counting Kit-8 assays, Transwell assays, colony formation assays, flow cytometry

Posted Date: January 20th, 2021

DOl: https://doi.org/10.21203/rs.3.rs-148995/v1

License: (c) (i) This work is licensed under a Creative Commons Attribution 4.0 International License. Read Full License 


\section{Abstract \\ Background}

Glioma is one of the most frequently occurring and lethal primary malignant tumors. MicroRNAs (miRNAs) are a newly identified modulator involved in the formation and progression of glioma.

\section{Methods}

Stable knockdown or over-expression of miR-548ac in U87 glioblastoma multiforme (GBM) cells was used to explore the function of miR-548ac in the expression of Wnt5a. Luciferase reporter constructs were used to investigate the correlation between Wnt5a expression and miR-548ac. Cell Counting Kit-8 assays, Transwell assays, colony formation assays and flow cytometry were used to investigate the functions of Wnt5a and miR-548ac in glioma. Xenograft experiments were used to evaluate tumor growth and metastasis in vivo. Western blotting was used to assess the function of the Wnt/ $\beta$-catenin pathway.

\section{Results}

miR-548ac overexpression down-regulated the proliferation, migration and invasion of GBM cells. In addition, knockdown of miR-548ac promoted expression of Wat5a, a direct target of miR-548ac, which plays an oncogenic role in glioblastoma stem cells. Reduced Wan5a expression partially rescued the miR548ac-induced enhancement of GBM cell proliferation, migration and invasion. Kaplan-Meier curve analysis revealed that high miR-548ac expression was associated with poor prognosis of patients with GBM.

\section{Conclusions}

miR-548ac suppresses GBM progression by targeting Wan5a. The miR-548ac/Wnt5a axis may be a new potential strategy for glioma therapy.

\section{Introduction}

Glioma is one of the most frequently occurring and most lethal primary malignant tumors [1]. The highly invasive growth pattern and resistance to surgical intervention (tumor resection), radiotherapy and chemotherapy results in a dismal prognosis for patients with glioma [2, 3]. Therefore, understanding the mechanisms underlying the progression of this complicated disease is important to enable the development of new treatment methods.

MicroRNAs (miRNAs) are a small class of highly conserved non-coding RNAs that regulate gene expression by binding the $3 \otimes$ untranslated regions (UTRs) of target mRNAs [4-6]. They play key roles in 
the regulation of gene expression [7]. MiRNAs have been reported to be involved in carcinogenesis of a variety of human cancers $[8,9]$. miR-548 is a large and poorly conserved primate-specific miRNA gene family consisting of 68 members, several of which have been reported to be associated with cancer [10, 11]. miR-548ac participates in the proliferation, migration and invasion of lung cancer and breast cancer $[12,13]$.

The purpose of this study was to explore the molecular mechanism underlying the development of glioma and to identify biomarkers for diagnosis and treatment of glioma. RT-qPCR and analysis of The Cancer Genome Atlas (TCGA) database were used to demonstrate that Wan5a is overexpressed in glioma. The carcinogenic effect of Wan5a and the anticancer effect of miR-548ac were then investigated through in vitro and in vivo assays (Cell Counting Kit-8 (CCK-8) assays, colony formation assays, flow cytometry assays, western blotting and tumor xenograft assays). miR-548ac was found to bind the 3هUTR of Wan5a, inhibit Wan5a expression and further suppress GBM progression.

\section{Methods}

\section{Cell culture}

The U87 human glioma cell line was purchased from the Chinese Academy of Medical Sciences (Beijing, China). U87 glioma cells were cultured in Dulbecco's modified Eagle's medium/high glucose with 10\% fetal bovine serum (Gibco, Carlsbad, CA, USA) at $37^{\circ} \mathrm{C}$ in $5 \% \mathrm{CO}_{2}$.

\section{Cell transfection}

An miR-548ac mimic, miR-548ac inhibitor and their related negative controls were transfected into cells (RiboBio Co. Ltd, Guangzhou, China). siRNA against Wan5a and siRNA control (siRNA NC) were purchased from GenePharma (Shanghai, China). For over-expression of Wnt5a, the sequence of Wnt5a was amplified by PCR and then cloned into plasmids. The U87 cells were then stably transfected with NC, miR-548ac and Wan5a plasmids, and oligonucleotides, with Lipofectamine 3000 (Invitrogen, CA, USA).

\section{Flow cytometry}

For cell cycle analysis, cells were fixed with $75 \%$ ethanol and resuspended in PBS. Thereafter, $500 \mu \mathrm{l} / \mathrm{ml}$ propidium iodide was added to the cells and incubated for another $30 \mathrm{~min}$ at room temperature, and labeled cells were detected with a flow cytometer (BD Biosciences, NJ, USA). The distribution of cells in G1, S and G2 phases was established with ModFit LT software (Verity Software House).

\section{Cell proliferation assays}

Cell proliferation was measured with CCK-8 assays in accordance with the manufacturer's instructions (Beyotime Institute of Biotechnology, Jiangsu, China). In brief, U87 cells were seeded in 96-well plates at 3000 cells/well. CCK8 solution $(10 \mu \mathrm{l})$ was added and incubated for $2 \mathrm{~h}$ at $37^{\circ} \mathrm{C}$. The absorbance was evaluated at $450 \mathrm{~nm}$ with a SpectraMax M5 microplate reader (Molecular Devices, CA, USA). 


\section{Colony formation assays}

U87 cells in growth phase were seeded at 1000 cells per well in culture medium. The cells were grown for 2 weeks at $37^{\circ} \mathrm{C}$, then fixed in $4 \%$ paraformaldehyde for $10 \mathrm{~min}$. Cell colonies were stained with $0.5 \%$ crystal violet for $30 \mathrm{~min}$ at room temperature and washed in water.

\section{Transwell invasion assays}

Transwell assays were performed to detect cell invasion. Cells were transfected for $48 \mathrm{~h}$, and then $2 \times 10^{5}$ cells were plated in the upper chamber (with Matrigel for invasion assays) of a 24-well Transwell plate, and $600 \mu \mathrm{l}$ of fetal bovine serum was added to the lower chamber. After incubation for $24 \mathrm{~h}$, cells on the upper surface were removed, and cells migrating to the lower chamber were fixed with $4 \%$ paraformaldehyde and stained with $0.1 \%$ crystal violet. For each experiment, cells were counted in six random fields.

\section{Wound healing assays}

Wound healing assays were performed to analyze cell migration. U87 cells at $5 \times 10^{5}$ cells per well were seeded in six-well plates and grown to confluency. A linear wound was made by scaping a pipette tip across the confluent cell layer, which was then washed twice to remove detached cells and debris. The size of the wounds was then observed and measured at the indicated times.

\section{RNA extraction and quantitative reverse transcription- polymerase chain reaction (qRT-PCR) analysis}

Total RNA was isolated with TRIzol reagent (Invitrogen) in accordance with the manufacturer's instructions and reverse transcribed to cDNA with the SuperScript ${ }^{\text {TM }}$ III First-strand Synthesis System (Thermo Fisher Scientific, MA, USA). For qRT-PCR analysis, the relative quantification of Wnt5a expression was computed with the comparative CT method $(\triangle \triangle C T)$.

Wnt5a forward primer: 5'-TCGACTATGGCTACCGCTTT3', reverse primer: 5'-CACTCTCGTAGGAGCCCTT-3'; miR-548ac forward primer: 5'-CAAAAACCGGCAATTACTTTTG-3', reverse primer: 5'CTCAACTGGTGTCGTGGA-3';

GAPDH forward primer: 5'-ATTCCATGGCACCGTCAAGGCTGA-3', reverse primer: 5'TTCTCCATGGTGGTGAAGACGCCA-3'.

\section{In vivo assays}

Lentivirus encoding Wnt5A and a non-targeting sequence (negative control, NC) were transfected into U87 cells to generate stably transfected cells. For assessing tumor growth in vivo, BALB/c nude mice were randomly distributed into three groups ( $n=6$ per group). LV-NC U87 cells $\left(1 \times 10^{6}\right)$, LV-siWnt5a U87 cells $\left(1 \times 10^{6}\right)$ and LV-miR-548ac + sh-Wnt5a cells $\left(5 \times 10^{6}\right)$ were separately resuspended in $150 \mu \mathrm{l}$ PBS and then 
subcutaneously inoculated into the flanks of BALA/c nude mice. The tumors were verified regularly at the indicated time points.

\section{Luciferase reporter assays}

Potential miR-548ac binding sites in the Wnt5A 3'UTR were predicted with the bioinformatics tools miRwalk (http://starbase.sysu.edu.cn/), miRDB (http://mirdb.org/) and TargetScan (http://www.targetscan.org/). The sites of the predicted target gene and a mutant variant were synthesized and cloned in the pmirGLO Dual-Luciferase miRNA target expression vector (GenePharma, Shanghai, China). U87 cells were seeded in 96-well plates and co-transfected $12 \mathrm{~h}$ later with plasmids or miR-548ac mimics with Lipofectamine 3000. The cells were harvested $48 \mathrm{~h}$ after transfection, and firefly and Renilla luciferase activity was analyzed with the Dual-Luciferase ${ }^{\mathrm{TM}}$ Reporter Assay System (Promega, Madison, WI, USA), according to the manufacturer's instructions.

\section{Western blot analysis}

Cells were homogenized with RIPA buffer (Thermo, MA, USA) and isolated on ice. Proteins were separated by SDS-PAGE and transferred to polyvinylidene difluoride membranes. After blocking with $5 \%$ skim milk, the PVDF membranes were incubated with primary antibodies. Antibodies against p-ERK1/2, total ERK1/2, total RAF, p-RAF, Wnt5a and $\beta$-actin were purchased from Abcam.

\section{Statistical analysis}

Prism 6 statistical software was used for statistical analysis. All data are presented as the mean \pm standard error of mean (SEM) from at least three independent replicates. Statistical analysis of data was performed with Student's t-test. Differences were considered statistically significant at $P<0.05$.

\section{Results}

\section{Overexpression or silencing of miR-548ac promotes or inhibits the proliferation of GBM cells}

miR-548ac was overexpressed and inhibited in U87 GBM cell lines to examine the function of miR-548ac in GBM proliferation and migration. RT-PCR was used to detect the transfection efficiency of miR-548ac. The results revealed that miR-548ac expression significantly increased or decreased after cells were transfected with miR-210-3p mimic or inhibitor, respectively, as compared with NC mimic or inhibitor. (Fig. 1A). Ki67 assays showed that miR-548ac overexpression significantly decreased U87 cell proliferation, whereas the miR-548ac inhibitor had the opposite result (Fig. 1B, C). In agreement with this finding, CCK8 assays showed that proliferation was greatly reduced when miR-548ac was overexpressed but increased when miR-548ac was inhibited (Fig. 1D). We then analyzed the cell cycle distribution and evaluated the proliferation of U87 cells by using fluorescence-activated cell sorting (FACS) after miR548 ac overexpression and inhibition. miR-548ac overexpression blocked the progression of the cell cycle in U87 cells, and the number of cells in G1 phase increased, whereas the number of cells in S and G2 
phases decreased. When miR-548ac was inhibited, the number of cells in G1 phase decreased, and the number of cells in S phase increased (Fig. 1E, F). Colony formation assays showed that miR-548ac overexpression resulted in a decrease in the number of colonies, thus indicating that miR-548ac inhibited U87 cell proliferation (Fig. 1G, H). These results indicated that miR-548ac inhibits GBM progression and consequently might function as a tumor suppressor of GBM.

Figure 1. miR-548ac upregulation or knockdown affects GBM cell proliferation in vitro. (A) qRT-PCR analysis of miR-548ac expression in U87 cells transfected with miR-548ac mimics, mimic control, miR548 ac inhibitor or inhibitor control. (B) Ki67 assay of cellular proliferation in U87 cells transfected with miR-548ac mimics or miR-548ac inhibitor. Bar $=200 \mu \mathrm{m}$. (C) Quantification of the percentage of Ki67positive cells. (D) CCK8 assay of cellular proliferation in U87 cells transfected with miR-548ac mimic or mimic control, miR-548ac inhibitor or inhibitor control. (E, F) FACS measurement of cell cycle distribution and quantification of the percentage of cells in $G 1, S$ or $G 2$ stages. $(G, H)$ Colony formation assay of cellular proliferation with U87 cells transfected with miR-548ac mimic or mimic control, miR-548ac inhibitor or inhibitor control. ${ }^{*} \mathrm{P}<0.05,{ }^{*} \mathrm{P}<0.01$, $* \star * \mathrm{P}<0.001$.

\section{Overexpression or silencing of miR-548ac promotes or inhibits the migration and invasion of GBM cells}

To assess the effects of miR-548ac on cell migration and invasion, we performed wound healing and Transwell assays with miR-548ac mimic or miR-548ac inhibitor. The wound healing assays showed that the migration of the U87 cells significantly decreased after miR-548ac overexpression (Fig. 2A, B, C). Transwell assays used to analyze the invasion of cells after miR-548ac overexpression indicated that increased miR-548ac expression decreased invasion by U87 cells (Fig. 2D, E). These results indicated that miR-548ac may affect the invasion and migration of U87 cells.

Figure 2. miR-548ac overexpression inhibited the migration and invasion of GBM cells in vitro. $(A, B$ and C) Cellular migration in U87 cells was determined with wound healing assays. Wound healing analysis $24 \mathrm{~h}$ and $48 \mathrm{~h}$ after transfection and quantification of the percentage of wound recovery. Bar $=400 \mu \mathrm{m}$. (D, E) miR-548ac overexpression reduced the rate of invasion by $\mathrm{U} 87$ cells, and miR-548ac knockout promoted invasion. Bar $=400 \mu \mathrm{m}$. ${ }^{*} \mathrm{P}<0.05,{ }^{*} \mathrm{P}<0.01, * \star * \mathrm{P}<0.001$.

\section{miR-548ac regulates Wnt5a expression}

The above results indicated that miR-548ac has remarkable effects on the biological behaviors of GSCs, but the underlying molecular mechanisms remain unclear. The bioinformatics databases Targetscan, miRwalk and miRBD suggested that several genes might be downstream targets of miR-548ac (Fig. 3A). A total of 11 downstream molecules associated with tumorigenesis were screened with KEGG analysis, including Wnt5a (Fig. 3B). Wnt5a facilitates tumor cell proliferation and migration and recruits macrophages for infiltration, and it is also highly expressed in tumor tissues[14] (Fig. 3C). We found that miR-548ac directly binds the 3'UTR of Wnt5a mRNA (Fig. 3D). We cloned the full-length 3'-UTR (wt/mut) of Wnt5a mRNA containing wild type (wt) or mutant (mut) sequences and inserted it into a luciferase 
reporter vector downstream of the firefly luciferase gene. When the possible binding sites were mutated, the inhibitory effect of Wnt5a was completely blocked (Fig. 3E). In addition, qRT-PCR indicated that transfection with miR-548ac mimic inhibited Wnt5a expression, whereas transfection of miR-548ac inhibitor promoted Want5a expression (Fig. 3F). Western blotting further confirmed that miR-548ac overexpression inhibited Wnt5a protein expression (Fig. 3G). Therefore, these results demonstrated that miR-548ac binds the 3囚UTR of Wnt5a and inhibits its expression, and also confirmed a negative correlation between miR-548ac and Wnt5a expression.

Figure 3. MiR-548ac targets Wnt5a and negatively regulates its expression in U87 cells. (A) Identification of the downstream target gene by online database analysis. (B) Use of KEGG analysis to identify factors associated with tumorigenesis in Wnt5a downstream molecules. (C) Wnt5a overexpression in GBM tissues compared with normal tissues according to GEPIA. (D) The predicted binding site of miR-548ac in the Wnt5a 3'UTR. (E) Luciferase assay with co-transfection of wt or mut Wnt5a 3'UTR constructs together with miR-548ac or negative control plasmids. (F) RT-qPCR analysis of Wnt5a expression. (G) Western blot analysis and quantification of Wnt5a expression. ${ }^{*} \mathrm{P}<0.05$, $* * P<0.01,{ }^{*} * \mathrm{P}<0.001$.

\section{miR-548ac suppresses GBM proliferation through Wnt5a}

Given that miR-548ac bound the 3 $\triangle$ UTR of Wnt5a and suppressed GBM progression, we hypothesized that miR-548ac suppression of GBM progression might be mediated by Wnt5a. To validate this hypothesis, we restored Wnt5a before overexpressing miR-548ac in GBM cells (Fig. 4A) and analyzed the phenotypes. $\mathrm{KI} 67$ assays showed that Wnt5a restoration rescued the inhibitory effect of miR-548ac overexpression on GBM growth, whereas Wnt5a knockout decreased proliferation (Fig. 4B, C). The results of CCK8 assays further confirmed this conclusion (Fig. 4D). FACS was then conducted to analyze the cell cycle, and the results revealed that Wnt5a rescue increased the number of cells in S phase and restored the number of cells in $\mathrm{G} 1$ phase to a normal level (Fig. 4E, F). Colony formation assays indicated that when Wnt5a was knocked out, the number of colonies formed decreased, whereas colony expansion was restored when Wnt5a was rescued (Fig. 4G, H). These data thus indicated that miR-548ac suppresses GBM progression through Wnt5a, thus promoting the proliferation of tumor cells.

Figure 4. Knockdown of Wnt5a inhibits U87 cell proliferation, whereas Wnt5a rescues the effect of the miR-548ac mimic. (A) Ectopic Wnt5a expression restored its protein level in miR-548ac overexpressing U87 cells. (B and C) Ki67 assays performed $48 \mathrm{~h}$ after transfection (bar $=200 \mu \mathrm{m}$ ) and quantification of the percentage of Ki67-positive cells (D) CCK8 assays indicated that Wnt5a overexpression restored the proliferation of miR-548ac-overexpressing U87 cells. (E, F) Cell cycle analysis conducted by flow cytometry and quantification of the percentage of cells in $G 1, S$ or $G 2$ stages. $(G, H)$ Colony formation assays indicated that Wnt5a overexpression restored the proliferation of miR-548ac-overexpressing U87 cells. ${ }^{*} \mathrm{P}<0.05,{ }^{*} \mathrm{P}<0.01, * * * \mathrm{P}<0.001$.

\section{miR-548ac suppresses migration and invasion of GBM through Wnt5a}


We restored Wnt5a expression before overexpressing miR-548ac in GBM cells to analyze the effects on migration and invasion. Wound healing assays showed that the migration of the U87 cells was significantly reduced after Wnt5a knockout, whereas Wnt5a rescued the migratory behavior (Fig. 5A, B). In addition, Transwell assays revealed that a decrease in the expression of Wnt5a impaired the invasive ability of GSCs, whereas Wnt5a rescued the impaired invasion of GSCs induced by miR-548ac overexpression (Fig. 5C, D). These results revealed that Wnt5a mediates the tumor-suppressive effects of miR-548ac when overexpressed in GSCs.

Figure 5. Knockdown of Wnt5a inhibits U87 cell migration, whereas Wnt5a rescues the effect of the miR$548 \mathrm{ac}$ mimic. (A, B) Wound healing analysis $24 \mathrm{~h}$ and $48 \mathrm{~h}$ after transfection (bar $=400 \mu \mathrm{m}$ ) and quantification of the percentage wound recovery. $(C, D)$ Transwell assays demonstrated the migration of U87 cells transfected with siWnt5a or miR-548ac mimic plus Wnt5a. Bar $=400 \mu \mathrm{m}$. Quantification of migrated cells is shown. ${ }^{*} \mathrm{P}<0.05, * * * \mathrm{P}<0.001$.

\section{Downregulation of miR-548ac expression activates the ERK/AKT pathway by targeting Wnt5a}

Wnt5a has been demonstrated to be a key factor in the development and progression of various cancers [15]. Wnt5a has been shown to activate NF-KB pathway-dependent survival signaling in tumor cells [16] and to promote the formation of blood vessels in tumor tissues [14]. Here, we investigated whether the ERK/AKT pathway might be involved in miR-548ac/Wnt5a signaling in GBM cells. Therefore, we determined the status of the ERK and AKT oncogenic signaling pathways in GBM cells. Cellular levels of p-ERK1/2 and p-AKT markedly decreased in U87 cells stably overexpressing miR-548ac compared with control cells overexpressing miR-NC, whereas no statistically significant decrease in total ERK1/2 and AKT was observed (Fig. 6A). Enforced Wnt5a expression restored the ERK and AKT signaling pathways that were inhibited by miR-548ac overexpression. Deletion of Wnt5a abrogated ERK and AKT signaling activation in U87/anti-548ac GBM cells (Fig. 6A). Together these results indicate that inhibition of Wnt5a in GBM cells with low miR-548ac expression reverses malignant phenotypes in GBM.

\section{Wnt5a restoration combined with overexpression of miR- 548ac suppresses tumor growth in nude mice}

To further confirm the above findings, we used an in vivo tumor model. Implantation of cells with restored Wnt5a levels and with miR-548ac overexpression resulted in tumors of the same size as those in the miRNC group. In addition, tumors in the siWnt5a group were larger and had greater volumes than those in the other groups (Fig. 6B, C). Meanwhile, RT-qPCR (Fig. 6D) confirmed that the Wnt5a expression levels were significantly reduce in the siWnt5a group, thus indicating that Wnt5a knockout decreases tumorigenesis in vivo.

Figure 6. Downregulation of miR-129-5p expression activates AKT/ERK pathways by targeting Wnt5a, and miR-548ac suppresses glioma cell tumor growth in vivo. (A) Western blot analysis of p-AKT, total AKT, p-ERK1/2 and total ERK1/2 in U87/miR-NC and U87/miR-548ac cells co-transfected with empty 
vector or Wnt5a expression vector (three replicates per group, three independent experiments per group). GAPDH was used as the loading control. (B) Pictures of tumors obtained from mice transplanted with Lvcon, Lv-siWnt5a or Lv-miR-548ac + oeWnt5a. (C) Tumor weight. Error bars, SEM. (D) Relative mRNA expression of Wnt5a of tumors in different groups. Error bars, SEM.

\section{Discussion}

In recent years, much attention has been focused on the function of miRNAs in cell regulation, differentiation, apoptosis and angiogenesis $[17,18]$. miR-548ac has been reported to inhibit the growth of several tumor types. For example, miR-548ac participates in proliferation, migration and invasion in lung cancer and breast cancer $[12,13]$. miR-548ac has been suggested to be an oncogene, but the function and underlying mechanisms of miR-548ac in glioma have remained poorly understood.

The present research examined the role of miR-548ac during glioma development. Overexpression of miR-548ac in U87 cells suppressed cell proliferation and clonogenicity, and blocked the S-to-G2/M transition, whereas inhibition of miR-548ac promoted these activities. We also demonstrated that miR$548 \mathrm{ac}$ is a direct target of Wnt5a in dual-luciferase assays, thus suggesting that miR-548ac suppresses Wnt5a expression by directly binding its 3' UTR.

Wnt5a is an evolutionarily conserved non-canonical Wnt ligand that activates several non-canonical WNT signaling pathways [19]. Wnt5a plays an important role in tumor progression, and over-expression Wnt5a has been suggested to be involved in some cancers, including colon cancer, breast cancer and neuroblastoma [20-23]. We observed that miR-548ac mediated down-regulation of Wnt5a inactivates GBM cells and blocks cell-cycle progression.

In summary, we shed new light on the role of miR-548ac and its target gene, Wnt5a, in controlling the cell cycle and their potential implications in pathological processes in U87 cell lines. miR-548ac overexpression down-regulated the expression of Wnt5 and inhibited malignant biological behavior in glioma. These findings highlight the function of miR-548ac in U87 cells, offering new insights into the regulatory network of the cell cycle, which may provide a new target and new strategies for the treatment of glioma.

\section{Declarations}

\section{Authors' contributions}

WS and HNL designed the study; WS, HNL, XL and XTW performed the experiments and prepared the figures; WS contributed to drafting the manuscript. All authors read and approved the final manuscript.

\section{Acknowledgements}

Not applicable.

\section{Competing interests}


The authors declare that they have no competing interests.

\section{Availability of data and materials}

The datasets used and/or analyzed during the current study are available from the corresponding author on reasonable request.

\section{Consent for publication}

Not applicable.

\section{Ethics approval and consent to participate}

The study was approved by the Ethics Review Committee of Affiliated Hospital of Nantong University (Nantong, China). The animal study followed the Guidelines for the Animal Care and Use approved by Affiliated Hospital of Nantong University.

\section{Publisher's Note}

Springer Nature remains neutral with regard to jurisdictional claims in published maps and institutional affiliations.

\section{References}

1. Reifenberger, G., et al., Advances in the molecular genetics of gliomas - implications for classification and therapy. Nat Rev Clin Oncol, 2017. 14(7): p. 434-452.

2. Ostrom, Q.T., et al., CBTRUS statistical report: Primary brain and central nervous system tumors diagnosed in the United States in 2006-2010. Neuro Oncol, 2013. 15 Suppl 2: p. ii1-56.

3. Tso, J.L., et al., Bone morphogenetic protein 7 sensitizes 06-methylguanine methyltransferase expressing-glioblastoma stem cells to clinically relevant dose of temozolomide. Mol Cancer, 2015. 14: p. 189.

4. Meister, G., et al., Human Argonaute2 mediates RNA cleavage targeted by miRNAs and siRNAs. Mol Cell, 2004. 15(2): p. 185-97.

5. Ambros, V., The functions of animal microRNAs. Nature, 2004. 431(7006): p. 350-5.

6. Rouleau, S., et al., 3' UTR G-quadruplexes regulate miRNA binding. RNA, 2017. 23(8): p. 1172-1179.

7. Zhang, K.L., et al., MicroRNA-566 activates EGFR signaling and its inhibition sensitizes glioblastoma cells to nimotuzumab. Mol Cancer, 2014. 13: p. 63.

8. Calin, G.A. and C.M. Croce, MicroRNA signatures in human cancers. Nat Rev Cancer, 2006. 6(11): p. 857-66.

9. Esquela-Kerscher, A. and F.J. Slack, Oncomirs - microRNAs with a role in cancer. Nat Rev Cancer, 2006. 6(4): p. 259-69. 
10. Liang, T., L. Guo, and C. Liu, Genome-wide analysis of mir-548 gene family reveals evolutionary and functional implications. J Biomed Biotechnol, 2012. 2012: p. 679563.

11. Malzkorn, B., et al., Identification and functional characterization of microRNAs involved in the malignant progression of gliomas. Brain Pathol, 2010. 20(3): p. 539-50.

12. Wang, Z., et al., miR-548b-3p functions as a tumor suppressor in lung cancer. Lasers Med Sci, 2020. 35(4): p. 833-839.

13. Shi, Y., et al., MiR-548-3p functions as an anti-oncogenic regulator in breast cancer. Biomed Pharmacother, 2015. 75: p. 111-6.

14. Hu, B., et al., Epigenetic Activation of WNT5A Drives Glioblastoma Stem Cell Differentiation and Invasive Growth. Cell, 2016. 167(5): p. 1281-1295 e18.

15. MacRae, C.A. and R.T. Peterson, Zebrafish as tools for drug discovery. Nat Rev Drug Discov, 2015. 14(10): p. 721-31.

16. Lopez-Bergami, P. and G. Barbero, The emerging role of Wnt5a in the promotion of a proinflammatory and immunosuppressive tumor microenvironment. Cancer Metastasis Rev, 2020. 39(3): p. 933-952.

17. Mellis, D. and A. Caporali, MicroRNA-based therapeutics in cardiovascular disease: screening and delivery to the target. Biochem Soc Trans, 2018. 46(1): p. 11-21.

18. Qadir, M.I. and A. Faheem, miRNA: A Diagnostic and Therapeutic Tool for Pancreatic Cancer. Crit Rev Eukaryot Gene Expr, 2017. 27(3): p. 197-204.

19. Oishi, I., et al., The receptor tyrosine kinase Ror2 is involved in non-canonical Wnt5a/JNK signalling pathway. Genes Cells, 2003. 8(7): p. 645-54.

20. Liang, H., et al., Wnt5a inhibits $B$ cell proliferation and functions as a tumor suppressor in hematopoietic tissue. Cancer Cell, 2003. 4(5): p. 349-60.

21. Blanc, E., et al., Low expression of Wnt-5a gene is associated with high-risk neuroblastoma. Oncogene, 2005. 24(7): p. 1277-83.

22. Li, S., et al., IL-1beta mediates MCP-1 induction by Wnt5a in gastric cancer cells. BMC Cancer, 2014. 14: p. 480.

23. Leris, A.C., et al., WNT5A expression in human breast cancer. Anticancer Res, 2005. 25(2A): p. 731-4.

\section{Figures}


A
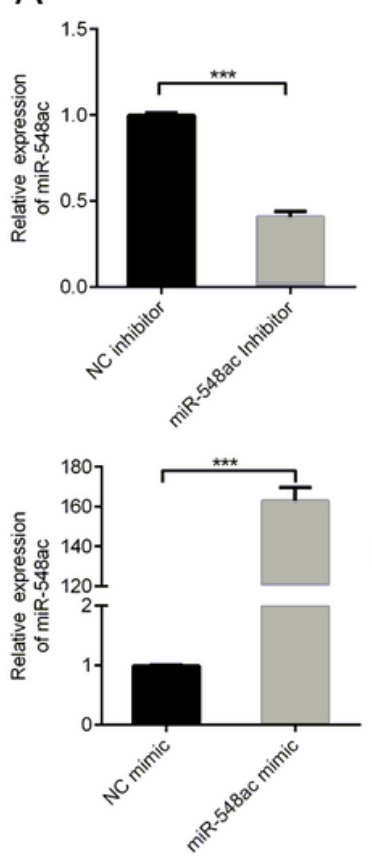

B

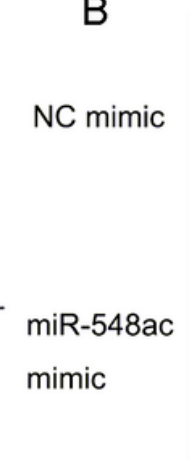

NC inhibitor
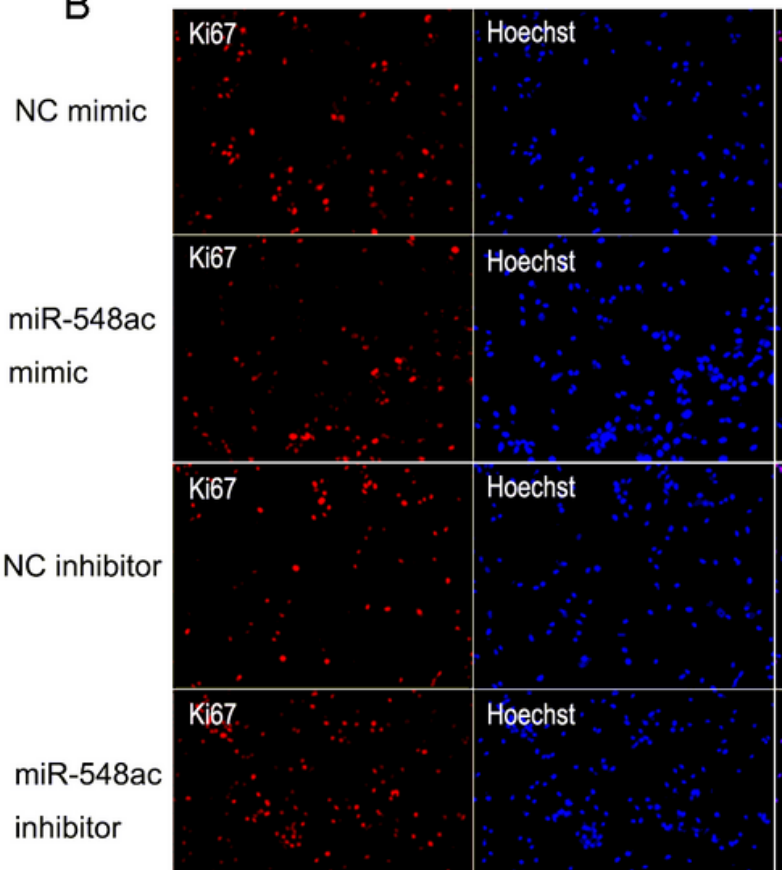

C

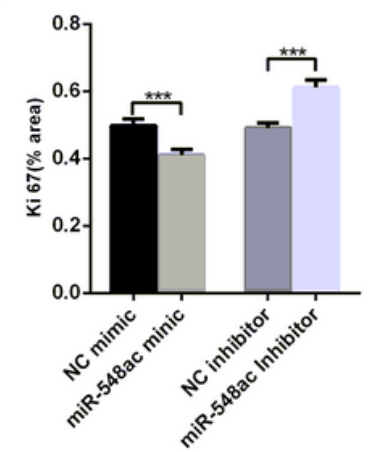

$\mathrm{D}$

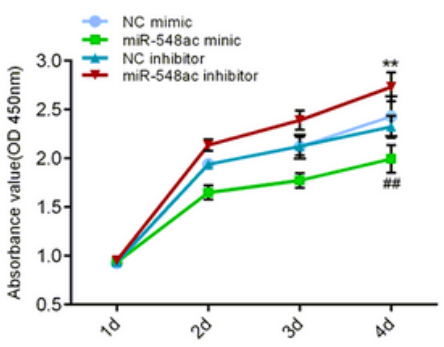

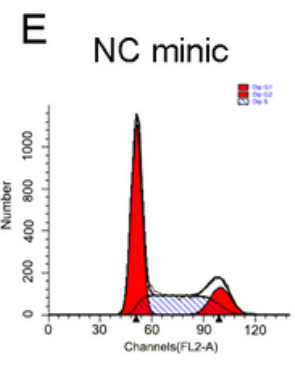

miR-548ac minic NC inhibitor

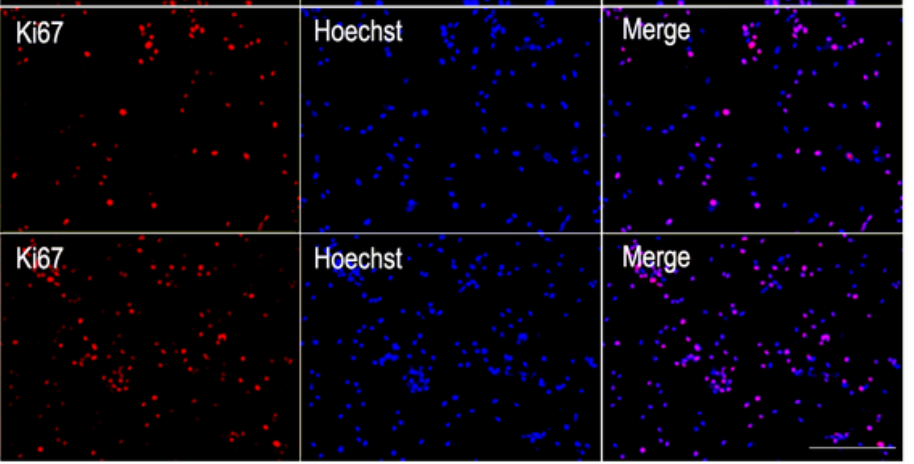

miR-548ac
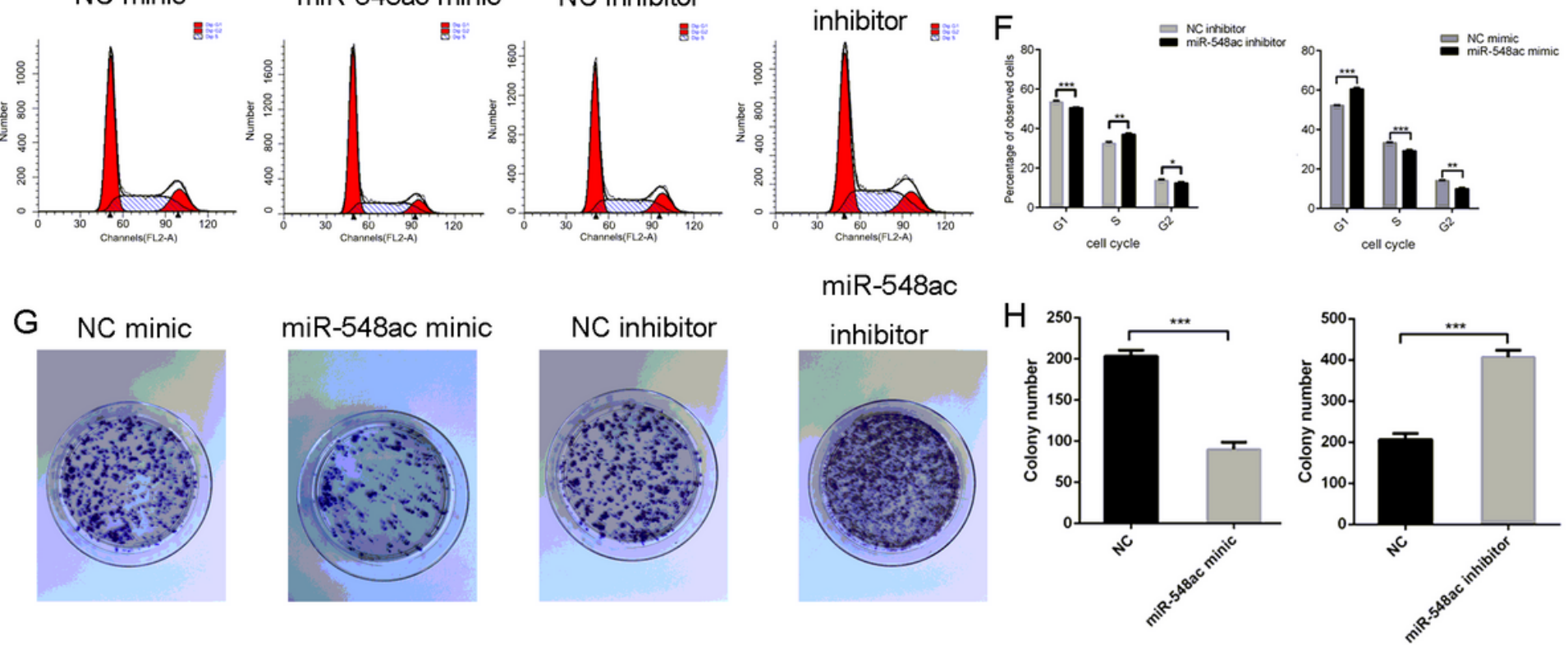

Figure 1

miR-548ac upregulation or knockdown affects GBM cell proliferation in vitro. (A) qRT-PCR analysis of miR-548ac expression in U87 cells transfected with miR-548ac mimics, mimic control, miR-548ac inhibitor or inhibitor control. (B) Ki67 assay of cellular proliferation in U87 cells transfected with miR$548 \mathrm{ac}$ mimics or miR-548ac inhibitor. Bar $=200 \mu \mathrm{m}$. (C) Quantification of the percentage of Ki67-positive cells. (D) CCK8 assay of cellular proliferation in U87 cells transfected with miR-548ac mimic or mimic control, miR-548ac inhibitor or inhibitor control. (E, F) FACS measurement of cell cycle distribution and quantification of the percentage of cells in $\mathrm{G} 1$, S or $\mathrm{G} 2$ stages. $(\mathrm{G}, \mathrm{H})$ Colony formation assay of cellular 
proliferation with U87 cells transfected with miR-548ac mimic or mimic control, miR-548ac inhibitor or inhibitor control. ${ }^{*} \mathrm{P}<0.05,{ }^{*} \mathrm{P}<0.01$, ${ }^{\star *} \mathrm{P}<0.001$.
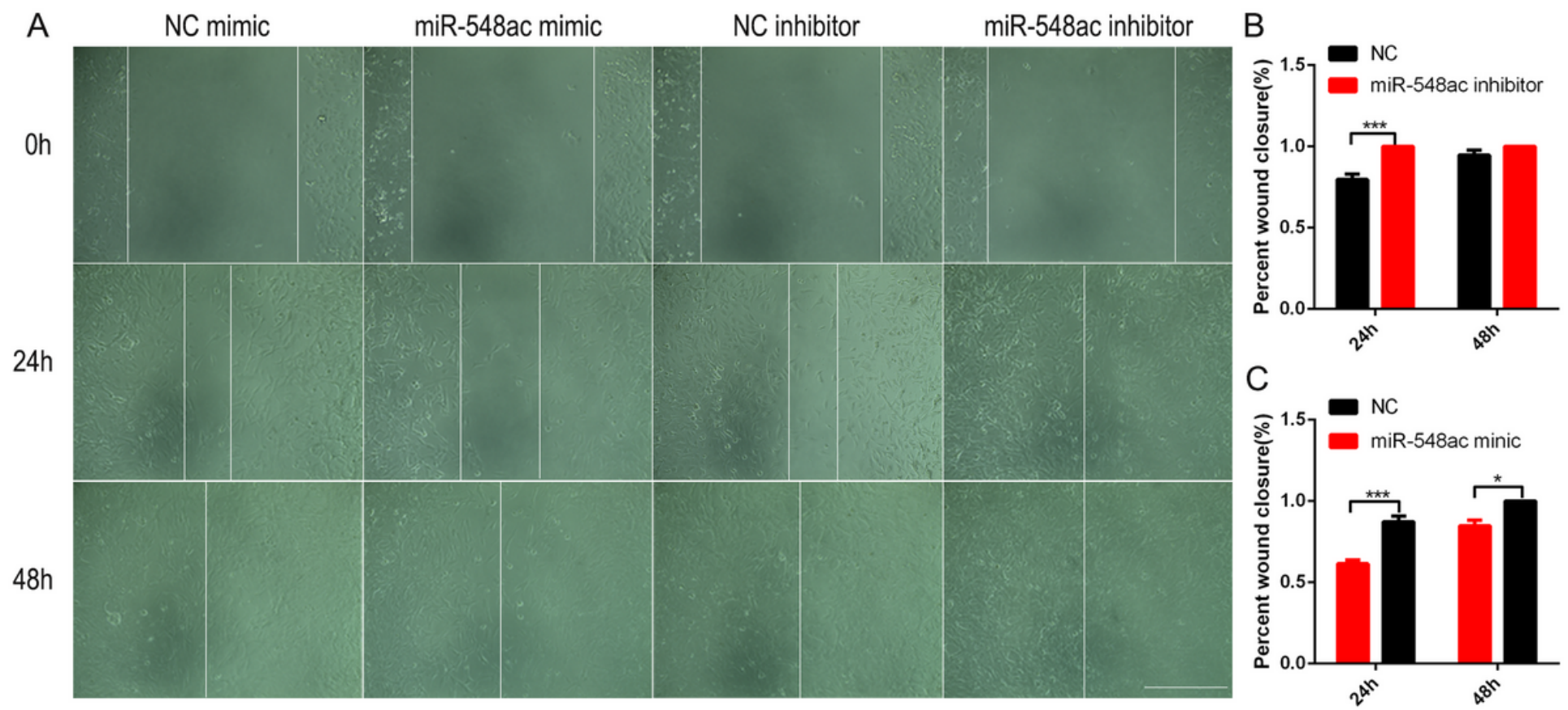

D
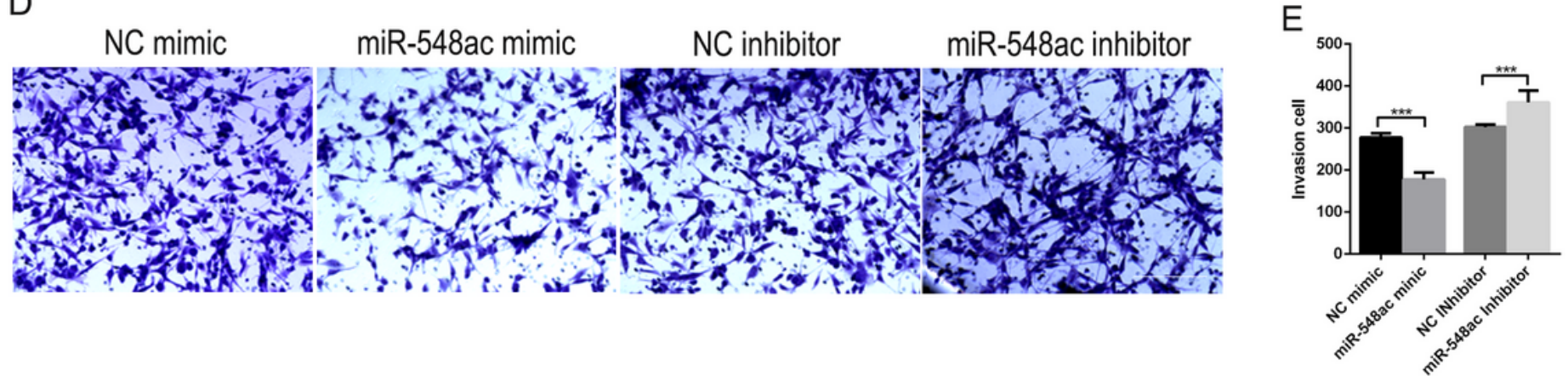

Figure 2

miR-548ac overexpression inhibited the migration and invasion of GBM cells in vitro. (A, B and C) Cellular migration in U87 cells was determined with wound healing assays. Wound healing analysis $24 \mathrm{~h}$ and 48 $h$ after transfection and quantification of the percentage of wound recovery. Bar $=400 \mu \mathrm{m}$. (D, E) miR548 ac overexpression reduced the rate of invasion by U87 cells, and miR-548ac knockout promoted invasion. Bar $=400 \mu \mathrm{m} .{ }^{*} \mathrm{P}<0.05, * * \mathrm{P}<0.01, * \star * \mathrm{P}<0.001$. 
A

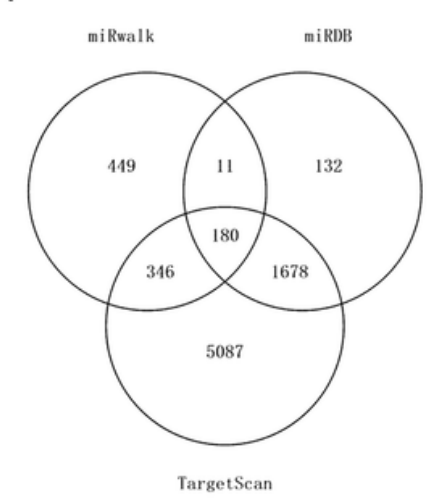

B

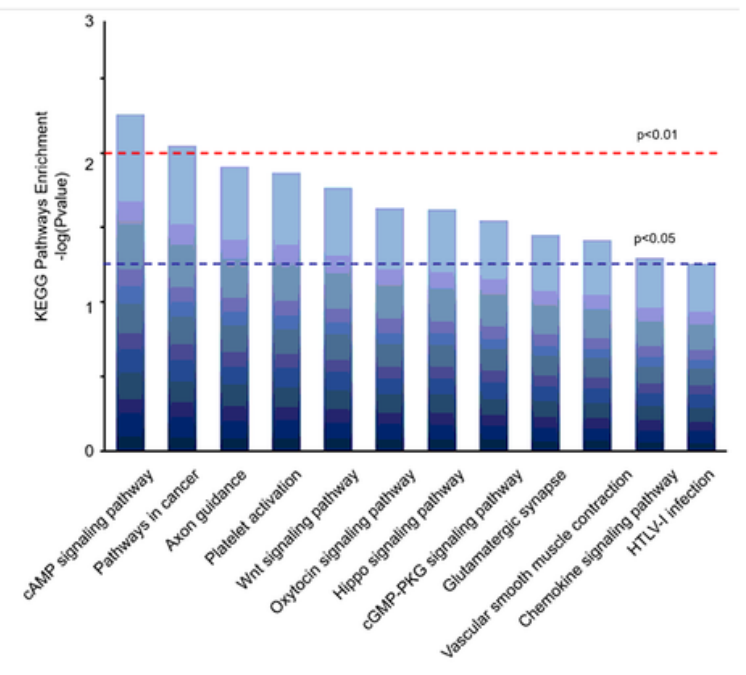

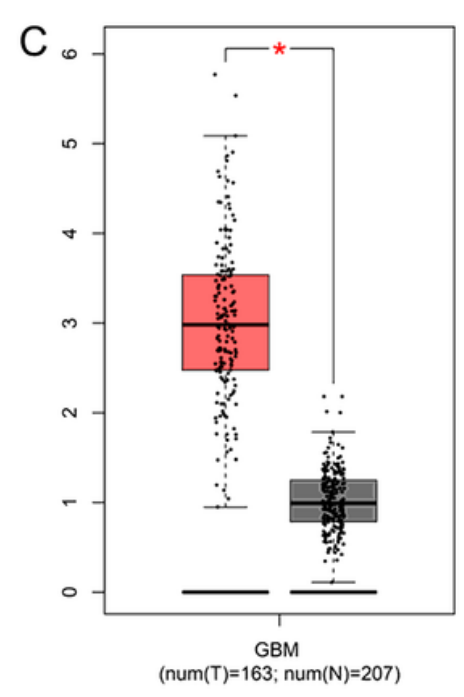

D

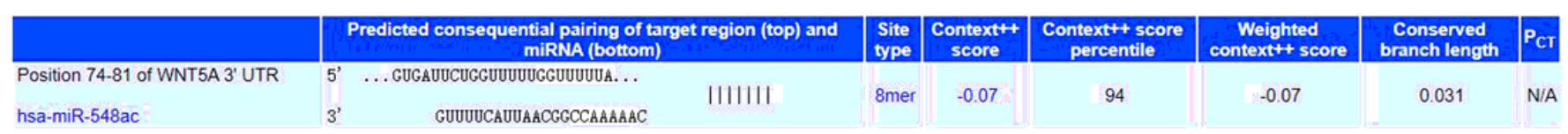

E

F

G
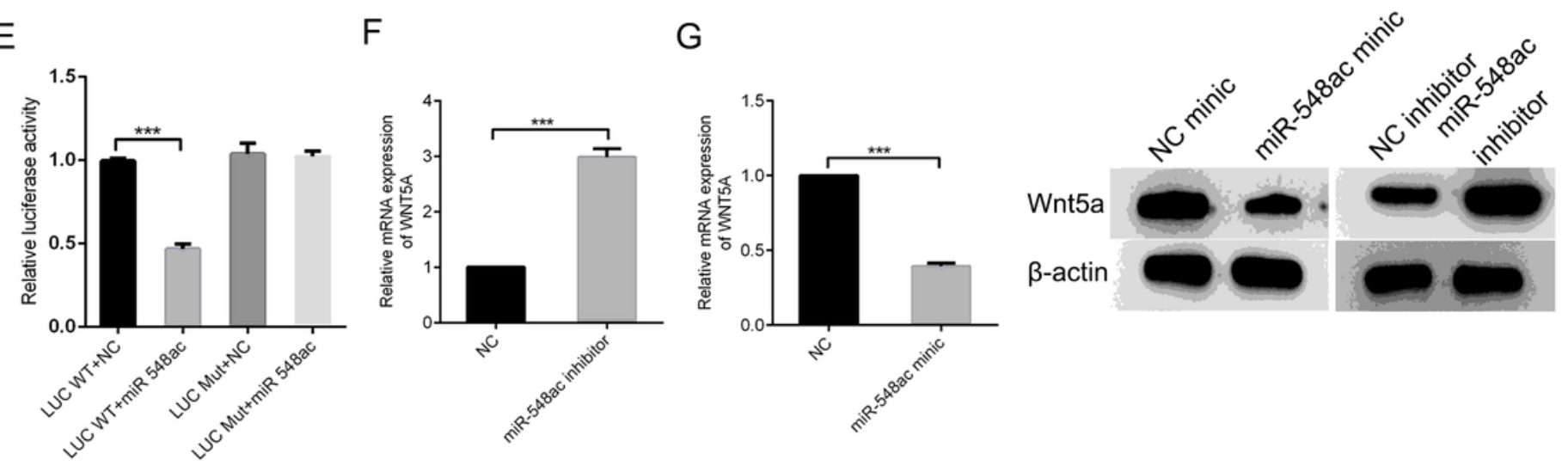

\section{Figure 3}

MiR-548ac targets Wnt5a and negatively regulates its expression in U87 cells. (A) Identification of the downstream target gene by online database analysis. (B) Use of KEGG analysis to identify factors associated with tumorigenesis in Wnt5a downstream molecules. (C) Wnt5a overexpression in GBM tissues compared with normal tissues according to GEPIA. (D) The predicted binding site of miR-548ac in the Wnt5a 3'UTR. (E) Luciferase assay with co-transfection of wt or mut Wnt5a 3'UTR constructs together with miR-548ac or negative control plasmids. (F) RT-qPCR analysis of Wnt5a expression. (G) Western blot analysis and quantification of Wnt5a expression. ${ }^{*} P<0.05,{ }^{*} P<0.01,{ }^{\star} * * P<0.001$. 

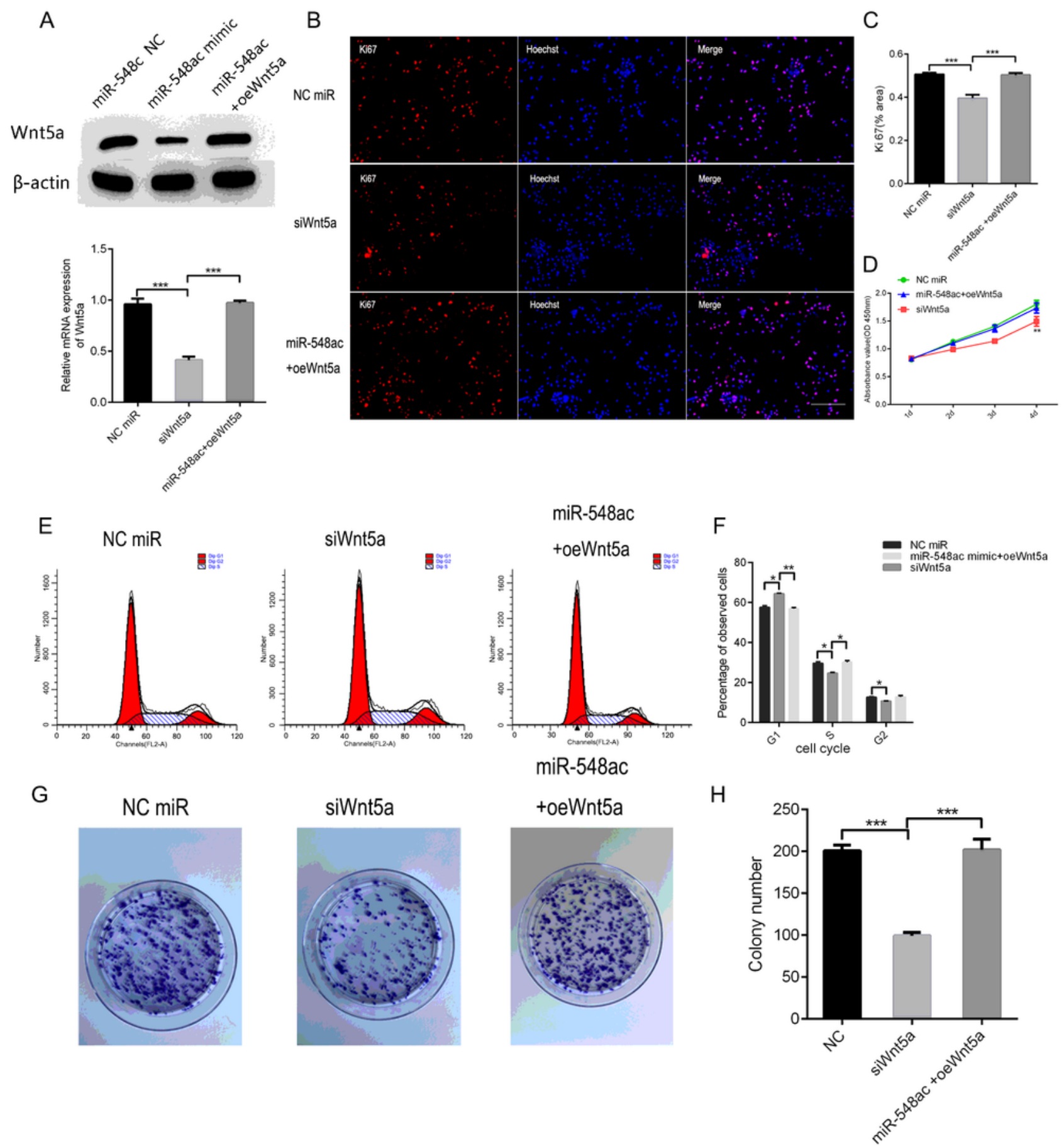

\section{Figure 4}

Knockdown of Wnt5a inhibits U87 cell proliferation, whereas Wnt5a rescues the effect of the miR-548ac mimic. (A) Ectopic Wnt5a expression restored its protein level in miR-548ac overexpressing U87 cells. (B and C) Ki67 assays performed $48 \mathrm{~h}$ after transfection (bar $=200 \mu \mathrm{m})$ and quantification of the percentage of Ki67-positive cells (D) CCK8 assays indicated that Wnt5a overexpression restored the proliferation of miR-548ac-overexpressing U87 cells. (E, F) Cell cycle analysis conducted by flow 
cytometry and quantification of the percentage of cells in $\mathrm{G} 1, \mathrm{~S}$ or $\mathrm{G} 2$ stages. $(\mathrm{G}, \mathrm{H})$ Colony formation assays indicated that Wnt5a overexpression restored the proliferation of miR-548ac-overexpressing U87 cells. ${ }^{*} \mathrm{P}<0.05,{ }^{*} \mathrm{P}<0.01,{ }^{*} * \mathrm{P}<0.001$.

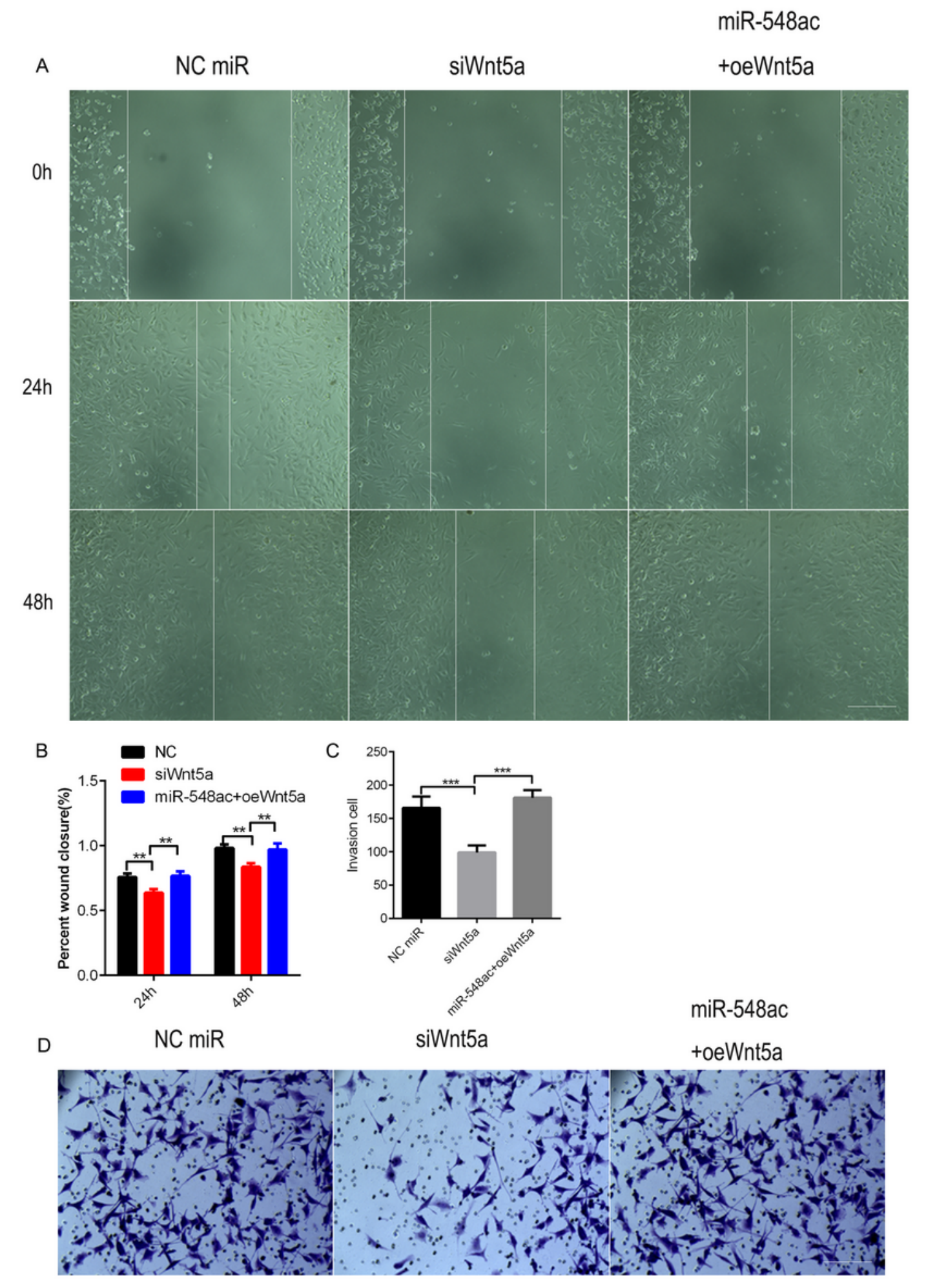

Figure 5

Knockdown of Wnt5a inhibits U87 cell migration, whereas Wnt5a rescues the effect of the miR-548ac mimic. (A, B) Wound healing analysis $24 \mathrm{~h}$ and $48 \mathrm{~h}$ after transfection (bar $=400 \mu \mathrm{m}$ ) and quantification 
of the percentage wound recovery. (C, D) Transwell assays demonstrated the migration of U87 cells transfected with siWnt5a or miR-548ac mimic plus Wnt5a. Bar $=400 \mu \mathrm{m}$. Quantification of migrated cells is shown. ${ }^{*} \mathrm{P}<0.05,{ }^{*} * \mathrm{P}<0.001$.
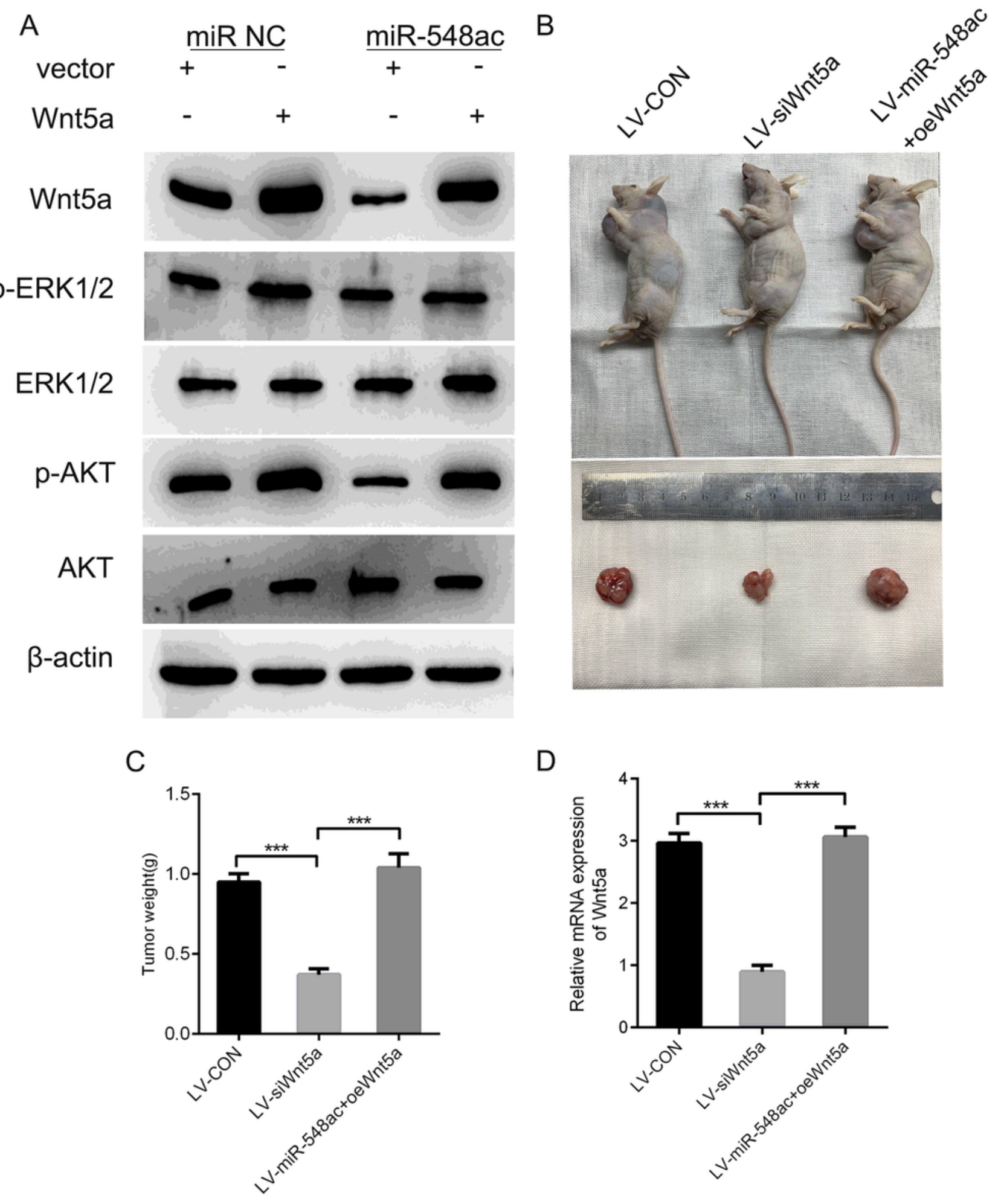

D

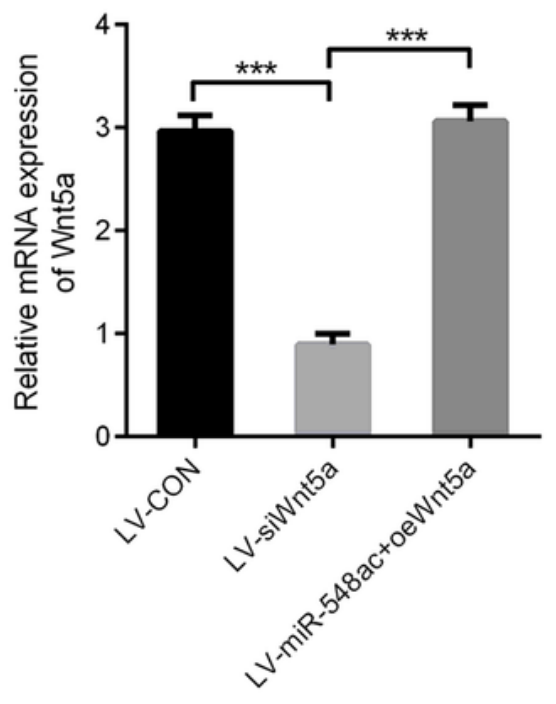

Figure 6

Downregulation of miR-129-5p expression activates AKT/ERK pathways by targeting Wnt5a, and miR548ac suppresses glioma cell tumor growth in vivo. (A) Western blot analysis of $p$-AKT, total AKT, p- 
ERK $1 / 2$ and total ERK1/2 in U87/miR-NC and U87/miR-548ac cells co-transfected with empty vector or Wnt5a expression vector (three replicates per group, three independent experiments per group). GAPDH was used as the loading control. (B) Pictures of tumors obtained from mice transplanted with LV-con, LVsiWnt5a or Lv-miR-548ac+oeWnt5a. (C) Tumor weight. Error bars, SEM. (D) Relative mRNA expression of Wnt5a of tumors in different groups. Error bars, SEM. 